\title{
EL DIÁLOGO COMO HERRAMIENTA PARA SOLUCIONAR CONFLICTOS TERRITORIALES SOBRE DESARROLLO ECONÓMICO Y SOCIAL EN LA ETAPA DEL POSCONFLICTO EN COLOMBIA
}

\author{
Martha Charris Balcázar ${ }^{1}$ \\ Ana Isabel Oñate Barros ${ }^{2}$
}

\section{Resumen}

Solidez para la paz en tiempos líquidos una posibilidad para construir democracia, El diálogo entre opuestos y su utilidad en la consolidación de una paz estable y duradera con un enfoque de desarrollo territorial y finalmente, la importancia de los Programas de Desarrollo con Enfoque Territorial en el desarrollo y progreso empresarial y de las comunidades, son los tres capítulos o enfoques de discusión que se proponen en adelante para evidenciar como el diálogo puede solucionar conflictos territoriales sobre desarrollo sostenible y liberar grandes tensiones entre empresas y comunidades.

$1 \quad$ Abogada de la Universidad Popular del Cesar (2013). Administradora Pública de la Escuela Superior de Administración Pública (2013). Magister de la Universidad de Zulia en Gerencia de Empresa (2017). Docente de la Universidad Popular del Cesar. Directora del Centro de Investigación y Documentación Socio jurídica de la Universidad Popular del Cesar. Docente investigadora y Tutora del Semillero de Investigación Acepciones del derecho. Email: marthacharris@unicesar.edu.co

$2 \quad$ Egresada del programa de Derecho de la Universidad Popular del Cesar. Fundadora y coordinadora académica del Centro de Derechos Humanos Rodrigo Uprimny 2016 - 2018. Segundo lugar en el Concurso Nacional De Derechos Humanos 2015. Premio a la mejor defensa de Estado escrita en idioma español por el American University Washington College of Law 2016. Juez en el segundo concurso de Derechos Humanos organizado por la Universidad de Cartagena 2017. Tutora del equipo Unicesar en el Concurso Nacional de Derechos humanos 2017 - 2018. Semillerista de investigación durante los años 2013 - 2017. Finalista del XVII concurso internacional de semilleros de investigación de Derecho procesal 2017. Email: anie702@hotmail.com 
Proponiendo entender este tipo de escenarios como una forma de sentar actores estratégicos en una gran discusión en la que se pueden llegar a acuerdos sin dejar de tener posiciones opuestas, en donde comunidades, institucionalidad y empresarios se escuchen a partir de unos puntos clave de convivencia en la búsqueda de soluciones efectivas, ello implica poner en marcha un proceso entre improbables que nos lleve a avanzar en medio de riesgos imprevisibles, hacia una sociedad fundamentada en la dignidad humana y la profundidad espiritual, en la búsqueda de la verdad sin miedos, en la superación de la exclusión social, económica y política; en la protección de la naturaleza y en la consolidación de instituciones justas, democráticas y creíbles. Y, por supuesto, en la lucha por terminar con la corrupción, que se fundamenta en la mentira, la codicia, el desprecio de los intereses generales y el engaño, tenemos derecho a ser diferentes, pero incluso esas diferencias hay que hacerlas sostenibles.

Palabras clave: Territorio - Desarrollo - Posconflicto - Diálogo

\section{Abstract}

Solidity for peace in liquid times (a possibility to build democracy), The dialogue between opposites and their usefulness in the consolidation of a stable and lasting peace with a focus on territorial development and finally, the importance of Development Programs with a Territorial Approach In the development and business and community development, are the three chapters or discussion approaches that are proposed in order to show how the dialogue can solve territorial conflicts on sustainable development and release great tensions between companies and communities. Proposing to understand this type of scenarios as a way to set strategic actors in a large discussion in which agreements can be reached while remaining in opposing positions, where communities, institutions and entrepreneurs are heard from a few key points of coexistence in the search for effective solutions, this implies starting a process between improbable that leads us to advance in the midst of unpredictable risks, towards a society based on human dignity and spiritual depth, in the search for truth without fear, in the overcoming social, economic and political exclusion; in the protection of nature and in the consolidation of just, democratic and credible institutions. And, of course, in the struggle to end corruption, which is based on lies, greed, contempt of general 
interests and deception, we have the right to be different, but even those differences must be made sustainable.

Key Words: Territory - Development - Postconflict - Dialogue

\section{Introducción}

Colombia 2020 y la Oficina del Alto comisionado para la paz han presentado recientemente una agenda de discusión denominada "diálogos entre opuestos", en donde se abordaron temas en relación a la Construcción de paz desde los territorios no solo desde la convivencia y la participación política, sino también desde el Desarrollo. La propuesta de diálogos entre opuestos en medio de sociedades divididas ha sido una estrategia diseñada inicialmente por el profesor estadounidense John Paul Lederach, y que se ha replicado en muchos países para establecer un diálogo sincero que ayude a superar conflictos armados históricos.

Esta mirada, entiende que el desarrollo en sí mismo es un diálogo entre opuestos y busca determinar ¿Cómo incorporar el trámite de conflictos a la institucionalidad? Y es que para ello debe existir un método, todo diálogo debe llevarnos a unos puntos específicos, a diagnosticar alertas tempranas de conflictos para intentar solucionarlos en la medida en que inician su evolución. La mayor parte de los conflictos son el resultado de conflictos antiguos que no se han resuelto anteriormente, la raíz de los conflictos está en la falta de participación de la sociedad, lo que el acuerdo nos dio fue un espacio en el cual identificamos los problemas, el acuerdo no los resolvió, pero si creó un espacio donde se podían empezar a discutir esos problemas.

Se pretende, con mucha humildad y con mucho respeto hacia las Víctimas y actores del conflicto en general, promover el diálogo que aún hace falta en los territorios, ahora que se está superando el dolor, para abordar un diálogo en donde se seleccionen temas que importen en lo local y lo departamental, que quizás nunca se han abordado con exhaustividad para mirar la totalidad de los datos, analizarlos desde distintos puntos de vista, establecer con rigor las diversas maneras de entenderlos y llegar, si es posible, a juicios compartidos sobre lo que podemos afirmar como realidad, independientemente de las expectativas, de los partidos y de las organizaciones a las que la sociedad libremente pertenezca, como lo decía 
el padre francisco de Roux en entrevista realizada por el tiempo en 2017 (DE ROUX, 2017).

Se desarrollan tres capítulos, el primero da cuenta de la importancia de promover espacios de participación democrática, a partir de sociedades sólidas y empoderadas, construir democracia a partir de educación, de un cambio en la cultura de la igualdad es el primer paso para poder desarrollar un gran diálogo, es quitarse la venda del miedo, de la estigmatización, es comprometer el "todos" en la construcción colectiva de País desde la pluralidad de actores y pensamientos, lo cual es vital para avanzar en una segunda etapa: dialogar.

Una segunda fase es entender la importancia del diálogo entre opuestos en la consolidación de una paz estable y duradera con un enfoque de desarrollo territorial, es promover conversaciones profundas y rigurosas entre las comunidades y las empresas para encontrar consensos en las dinámicas del desarrollo local, para que juntos fijen derroteros sobre su visón del territorio, a partir de la democracia y las líneas de convivencia establecidas.

Y por último, se expone como ya se están realizando algunos diálogos en el País desde los territorios y con actores estratégicos, promovidos por la misma institucionalidad y otros por iniciativa privada, pero además de eso, hay otros espacios de diálogo que deben generarse, que deben nacer desde la sociedad civil, y que tienen como reto generar la sostenibilidad de este nuevo proceso, pero sobretodo de llegar a consensos que se promuevan y defiendan.

\section{Solidez para la paz en tiempos líquidos, una posibilidad para construir Democracia}

Podría pensarse que ha ocurrido un error al escribir solidez en el título de este capítulo y que en su lugar quiso indicarse el término solidaridad, puesto que la paz indica ineludiblemente un sistema de valores sin los cuales esta no podría entenderse; sin embargo, ha sido intencional hacer un llamado a la solidez en tiempos líquidos como una de las muchas oportunidades que ofrece el postconflicto. La liquidez (en contraposición a la solidez), según BAUMAN (2007): 
Se caracteriza por revelar sociedades alimentadas por el miedo y la incertidumbre, donde las formas sociales parecen no poder mantenerse mucho tiempo, como si se descomponen y derriten antes de ser asimiladas, en contraposición, la solidez convierte cada una de esas barreras liquidas en un reto a superar, es decir en una posibilidad, entendiendo esta última como toda circunstancia que puede llegar a realizarse.

En el contexto actual, después de más de cincuenta años de conflicto, es casi imposible no intentar cuantificar el daño que ha dejado la guerra, un resquebrajado tejido social, un Estado ilegitimado, e incluso, la democracia como un sueño inconcluso; consecuencias fácticas que de mantenerse son un riesgo para sostener la paz, porque perpetuán en liquidez a la sociedad colombiana y lo que es peor aún a quienes fueron actores del conflicto marcada por el intento de permanencia del terror como forma de vida y estrategia para conquistar el poder, la resistencia a la solidaridad, la permanencia de la inequidad social y el debilitamiento de las instituciones del Estado. Estas marcas tan propias del hoy, no han sido distintas en toda la historia, incluso podría afirmarse que fueron la causa misma del conflicto, factores estos que son signos de inestabilidad social.

Por ello construir paz en medio del posconflicto, implica en primera medida, un cambio mental, porque cuando no se entiende que hay que mirar de otra forma el pasado, el presente y el futuro, la construcción de esta deviene en un imposible, puesto que los problemas estructurales no podrán solucionarse sin un cambio de lenguaje, porque la decir de LEVINÁS (1990), "la paz es un primer lenguaje, es el acontecimiento inicial del encuentro con el otro, como ese que es insustituible".

En segundo orden y no menos importante, para hacer exitoso el posconflicto deben tomarse las causas del conflicto y convertirlas en el plan de acción de las soluciones que necesita el país para solidificarlo, esto es, que si la segregación política, la inequidad, la ausencia de educación de calidad, el miedo colectivo y la inseguridad, la ausencia del Estado y la ineficiencia de sus instituciones fueron su causa, luego entonces, debe existir una oleada democrática que amplíe los espacios de participación de todos los sectores y de todos aquellos que no se identifiquen o no con algún colectivo, grupo o partido político, deben generarse oportunidades de empleo, dignificarse las condiciones de los trabajadores, apoyar al desarrollo científico, ampliar la 
cobertura de la educación pública y de calidad, superar la creencia de que la seguridad se logra a través de las armas, fortalecer los sistemas judiciales y la gobernabilidad del país, incluso, combatiendo desde los estamentos policivos la corrupción.

Bajo esta estructura mental, entonces es posible hablar de paz y la oportunidad única e histórica que en específico aporta el posconflicto, en lugar de simplemente ofrecerla: la de construir democracia y fortalecer la gobernabilidad, porque, el posconflicto comosuperación delos males sociales progresivamente permitirá la profundización de la democracia y esta a su vez, elementos que se muestran cruciales para lograr una construcción positiva como la deslegitimación de la guerra, el reconocimiento de la voluntad popular y del protagonismo de la ciudadanía, el funcionamiento de la justicia, la deliberación y la transformación social.

¿Pero una vez instaurada qué? Puede sobrevivir fácilmente o sostenerse, la respuesta es que sin gobernabilidad no, citando a REVEL (1983), "la democracia es ese régimen paradójico que ofrece a quienes quieren abolirla la posibilidad única de prepararse a ello en la legalidad", hacer la paz es más difícil que hacer la guerra o incluso que terminarla, por ello los retos no son otros que apostarle al posconflicto con sus múltiples, aunque costosas ventajas:

La primera de ellas, debe ser necesariamente, mejorar la calidad de la educación, la terminación del conflicto debe generar educación, blindando las decisiones democráticas que requiere el posconflicto puesto que si la estrategia será la desinformación, la mentira y la desacreditación para perpetuar el temor y la guerra con fines de poder, con educación de calidad es posible combatir la Pos verdad, para abrir los debates para evitar las famosas verdades absolutas y darle la bienvenida a las tesis, por ello los debates siempre estarán abiertos, para argumentos en contra o a favor, respetando las distintas posiciones máxime si no se comparten; pero pese a ello, la razón siempre nos indicará que es lo mejor; y debatir deliberadamente es una utilidad que solo puede darse si la educación es sólida y la inteligencia bien repartida.

El posconflicto debe permitirnos crear sociedades más éticas, la paz trasciende la esfera política, jurídica y hasta moral, la paz se logra a través de ética y la bondad, del reconocimiento al otro, no para obedecerlo 
sino para entenderlo como el otro, un ser que está ahí, que tiene derechos, para responderle, la ética no hace cuestionamientos arbitrarios, la ética perdona porque entiende razones así sean las contrarias a sus ideales, por eso apostarle a una sociedad o a generaciones que se imbriquen de valores como reconciliación que trae consigo el posconflicto es una apuesta única que genera sociedades equilibradas, tolerantes y sostenibles, puesto que la ética no se enseña, se muestra, y perdonar con la continuidad de una mejor vida es una forma de mostrar esa ética que se contagia en una sociedad que cree en la vida y que sueña con vivir bien, y que se concentra en trabajar para ello, apostándole al desarrollo de sus territorios, en lugar de continuar incentivando el odio.

La paz debe promover sociedades incluyentes e igualitarias, el enfoque de género es el reconocimiento de la igualdad de derechos entre hombres y mujeres y de las circunstancias especiales de cada uno. Supone reconocer que el conflicto ha impactado de manera diferenciada a la mujer y que, en consecuencia, se requieren acciones distintas y específicas para restablecer sus derechos en una etapa de posconflicto, en consecuencia la igualdad de género como consigna social toma un papel mucho más preponderante que el que ha tenido antes en la agenda nacional, adicionalmente brinda a miles de mujeres y de hombre cumplir su rol en la sociedad, y recuperar sus vidas, como madres, padres, esposos o hijos.

Desde estos tres enfoques de reconocimiento, se inicia una verdadera construcción de paz en los territorios, que permitirá avanzar hacia temas más gruesos, pero sobre los cuales es necesario sentarse a dialogar. Es necesario iniciar por darle una solidez moral, ética y social a quienes se invitan a sentar en ese gran diálogo territorial, para que ellos mismos a través de su sentir puedan finalmente descubrir que es lo que realmente quieren para sus comunidades, para su entorno, para luego discutir esos elementos esenciales del desarrollo, reconociendo que en los territorios aún hay una serie de conflictos como los relacionados con el uso del suelo, las industrias extractivas y los conflictos entre las comunidades indígenas, campesinas y tribales, entre la agroindustria y la pequeña economía campesina, la inadecuada priorización del gasto por la institucionalidad local, entre otros, esa es la verdadera democracia.

Tal como lo refiere IBAÑEZ (2018) "No hay solución perfecta a los problemas de desarrollo, no existen fórmulas únicas, es necesario construir 
una visión colectiva, existen distintos factores que deben tenerse en cuenta entre ellos la temporalidad, el diálogo es un proceso", en ese sentido los acuerdos y el desarrollo tiene tienen temporalidades muy diferente, y entonces el gran reto es cómo lograr que hay una asimetría en estos tiempos de transición, por ello lo primero es construir una visión colectiva y eso sólo es posible a través de un proceso de pacificación y sensibilización sobre el posconflicto y lo que ello representa.

\section{El diálogo entre opuestos y su utilidad en la consolidación de una paz estable y duradera con un enfoque de desarrollo territorial}

Las Naciones Unidas (ONU) han definido la justicia transicional como:

Toda la variedad de procesos y mecanismos asociados con los intentos de una sociedad por resolver los problemas derivados de un pasado de abusos a gran escala, a fin de que los responsables rindan cuentas de sus actos, servir a la justicia y lograr la reconciliación.(ONU, 2004, Párr. 8)

En el ámbito de la justicia y el derecho este término es muy frecuente y su discusión, se le asocia a la justicia restaurativa, redistributiva, adicionalmente al existir un alto componente de verdad se crean comisiones de la verdad y un andamiaje institucional que permite garantiza verdad, justicia, reparación y no repetición, luego entonces, se parte de la base que (i) no puede existir justicia transicional sin respeto por los derechos humanos (ii) existen derechos fundamentales que solo pueden ser garantizados en un sistema de transición y no en un estado de guerra o en ausencia de democracia.

La justicia transicional, tal como lo ha expuesto el secretario general de las naciones unidas abarca toda la variedad de procesos y mecanismos asociados con los intentos de una sociedad por resolver los problemas derivados de un pasado de abusos a gran escala, a fin de que los responsables rindan cuentas de sus actos, servir a la justicia y lograr la reconciliación. Tales mecanismos pueden ser judiciales o extrajudiciales y tener distintos niveles de participación internacional (o carecer por completo de ella) así, como abarcar el enjuiciamiento de personas, el resarcimiento, la búsqueda de la verdad, la reforma institucional, la investigación de antecedentes, la remoción del cargo o combinación de todos ellos. 
De lo anterior no se colige otra cosa más que deben existir cuatro elementos básicos de la noción de justicia transicional: (i) las medidas de transición o pacificación deben respetar un mínimo de justicia; (ii) amparar los derechos de las víctimas en cumplimiento del derecho internacional; (iii) puede existir flexibilidad en la aplicación de dichos estándares, es decir no hay un compendio taxativo de medidas solo deben cumplirse con los principios básicos de la justicia transicional, (iv) debe existir de manera cierta una transición con alcances políticos.

Ese concepto de lo transicional izado en la justicia ha llevado a establecer que existe un cambio social, político, económico e incluso cultural en Colombia, que justifica un cambio de paradigma en el interpretación de los principios constitucionales o generales del derecho. Repensar la transición como un hecho que constituye el avance social e histórico más grande de los últimos tiempos, necesariamente debe arrojar a establecer sí ello implica cambiar el modelo jurídico o las respuesta legal a los problemas. Ahora bien ¿Cómo es la transición de todo aquello que no es justicia, ni derecho? ¿Podemos extrapolar esos criterios de lo transicional de la justicia a otras esferas del posconflicto como los temas de Desarrollo territorial?

El diálogo en sí mismo es una transición, es la expresión más natural del respeto y garantía de los derechos humanos, es por ello que en lo territorial y en relación a aspectos de construcción de paz, sostenibilidad y empresa repensar cual es el territorio que se quiere tener y lograr que los diálogos lleven a acuerdos a las comunidades y las empresas es un elemento esencial. Ello requiere no sólo hablar con la verdad y aprender a escuchar a la sociedad civil mientras estos también aprenden a escucharse en medio de sus diferencias, que cuando se hable de empresa, de medio ambiente, de productividad, decompetitividad ello signifique obligatoriamente hablar de equidad y equilibrio social, proponer espacios en donde todos sean incluidos, un verdadero modelo de transformación es exitoso cuando hay equilibrio social y lucha contra la desigualdad, porque no hay nada sobre paz y derechos humanos que se pueda hacer desde la desigualdad, cuando no hay un compromiso de lucha contra la desigualdad en el corazón del modelo de competitividad.

Entender esa verdad enunciada, debe prometer un modelo de justicia social, de seguridad alimentaria, una reforma agraria que consolide esas expectativas, esas garantías, debe haber un cambio de mentalidad en la forma 
en como nos relacionamos con los territorios, que permita reflexionar sobre esos temas críticos que siguen generando tensiones entre las comunidades, y dialogarlos, discutirlos, para encontrar una manera de proponer soluciones, esa generación de oportunidades es una forma de justicia y a su vez de reparación.

A partir de esas premisas, debe crearse una institucionalidad que represente dichos valores colectivos y que esté acorde a esos acuerdos o consensos que la sociedad civil respaldó en un proceso dialógico que permitan continuar dialogando, al paralelo sobre las múltiples tensiones que existen, pero que a su vez permita ir desarrollando un modelo sostenible que genere progreso equitativamente, una apuesta que ha funcionado en algunos conflictos armados y sociales en otros países.

En Irlanda, por ejemplo, para superar el conflicto se creó una institucionalidad, un organismo con personal de todos los sectores: Un responsable de cultura, idiomas, alimentos, ambiente y otros, poco a poco esos organismos sectoriales pusieron en pie una estructura dinámica para empezar a afrontar los retos del desarrollo.

También se tuvo una disposición de recursos para la reconstrucción del tejido social, de 1994 hasta 1999, la Irlanda del posconflicto tuvo años de mucha desorganización, la solución fue diseñar unas plataformas locales o asociaciones estratégicas para exigir desde el punto de vista local sobre lo que ellas debían ponerse de acuerdo y así distribuirse los recursos. De todo lo anterior, resulto una sociedad civil muy empoderada y a el proceso de paz se le dio continuidad, porque esas comunidades están involucradas, esa fue la clave la superación del conflicto en Irlanda.

Por su parte, ESPIAU (2018), explica desde su experiencia en el País Vasco, que las transformaciones socioeconómicas en un contexto de violencia son factibles, para él, la dimensión de los conflictos es correlativa con los daños ocasionados, desde esa perspectiva propone cuatro elementos para poder producir un cambio en los entornos desde el diálogo:

1. Cambios en la dimensión cultural a partir del entendimiento de las narrativas en conflictos, lo que ha existido es una meta narrativa sobre puntosquenoestabanen cuestión, sobrelosquenose hablaba. Puede darse en una dimensión positiva o negativa, la primera nos lleva a una transformación y la segunda a un retroceso. Hay que 
empoderar a la sociedad para poder cumplir con los procesos de desarrollo.

2. Reapropiarse del concepto de competitividad. Se le huyen a estos debates sobre competitividad y desarrollo sostenible, la cuestión no es tanto como se distribuye la riqueza sino como crear riqueza de forma distribuida.

3. Incorporar la idea de la igualdad a la anterior sobre competitividad. Existe un sistema basado en la continuidad de la desigualdad o existe un modelo competitivo, hay que pensar en proyectos no lineales, lo que requiere de sus movimientos de transformación, hay que gobernar con ellos.

4. Se debe trabajar desde las dimensiones o dinámicas comunitarias, proponer nuevas herramientas de co-creación y co-gobernanza, ello debería permitir determinar sí los volúmenes de inversión han servido para transformar las realidades, es necesario replantear la priorización de las inversiones.

Ahora, es claro que no hay solución perfecta a los problemas de desarrollo, no existen fórmulas únicas, por ello es necesario construir una visión colectiva, existen distintos factores que deben tenerse en cuenta entre ellos la temporalidad, en este sentido los acuerdos y el desarrollo tienen temporalidades muy diferentes, y entonces el gran reto es cómo lograr una asimetría en estos tiempos de transición. Los diálogos deben conducir a algo, de no tener resultados hace que la gente se sienta traicionada y se decepcione. En Guatemala pasó, es muy difícil sentar a hablar a las personas, el diálogo es un camino largo que requiere transformaciones:

- En una dimensión personal. Un cambio en los mapas mentales de las relaciones interpersonales.

- Generar nuevas capacidades en las sociedades.

- Generar cambios institucionales que apoyen a los procesos de paz en los territorios.

El enfoque del territorio debe ser desde el desarrollo, no sólo de reconciliación. Hay que construir paz desde los territorios, escuchar las dinámicas locales y no la visión única desde lo nacional. La desigualdad 
estructural hay que superarla iniciando con las bases de los modelos de desarrollo, generando dificultades para el desarrollo muy serias.

Hay en algunos territorios una institucionalidad, es muy débil o en el peor de los casos inexistente. Pensar en estos escenarios incluyendo en la fórmula: comunidades + Estado + Empresarios; los territorios y los pequeños empresarios deben entrar allí., de ahí que la reconciliación tiene una dimensión ambiental, social y de desarrollo.

En Colombia, éstas experiencias y/o aprendizajes encajan muy bien, cuando la guerra inició se salía a las calles y no se veía al Estado, antes de ello era así y aún después de la guerra en algunos territorios no existe una institucionalidad que respalde al ciudadano en sus necesidades fundamentales.

DIEZ (2018), propone tres etapas del diálogo para superar grandes conflictos sociales que siguen latentes posterior a la firma de un acuerdo:

La primera, que es lo más difícil es hablar sobre la crisis, es decir el nombre, el color, la edad de la crisis, la segunda es hablar sobre el yo, ¿qué hizo la crisis conmigo? y la tercera es escuchar la voz del otro ¿Cómo te duele? ¿Qué fue lo que te pasó? ¿Cómo te impactó la crisis? escuchar al otro es la clave.

Cuando finalmente, se ha logrado dialogar sobre esos aspectos entonces es posible responder a la pregunta sobre ¿cuál es el territorio que queremos tener? Y a partir de esa respuesta establecer si cada una de las inversiones que se están dando desde lo local, son acorde a la visión común de futuro, o cómo se enmarca esa inversión en dicha visión. Hay una línea en donde confluyen los proyectos y las comunidades, desprendiéndose de ello existen unos imaginarios colectivos, de destrucción, de apatía, de mitos, de resignación, entre otros.

Por eso, el rol de las empresas también debe ser de una actitud permanente hacia el diálogo, de escuchar y promover la superación de conflictos. Las ideas para la paz han desarrollado una guía, desde la cual propone que las empresas y las fundaciones empresariales: 
Desde su gestión y mediante iniciativas concretas, inciden positivamente en la construcción de paz cuando contribuyen a generar condiciones sociales, políticas, ambientales y culturales que son inclusivas y justas. Aportan a la paz cuando construyen relaciones de confianza y promueven la solución pacífica de diferencias entre actores sociales, públicos y privados. Todo esto con un enfoque transversal de acción sin daño y conscientes de ser parte del territorio donde operan. Los aportes empresariales para lograr estas condiciones favorables a la paz no se limitan a las acciones que se puedan desarrollar en el marco de las políticas de Responsabilidad Social Empresarial (RSE) o desde las áreas de relacionamiento con comunidades o sostenibilidad. Por el contrario, son transversales a todas las áreas funcionales de la empresa y a todas sus esferas de influencia, desde la organización interna hasta las relaciones de negocio, las estrategias de mercado o la gestión de la cadena de valor. (FIP, 2016. Pág. 2)

Desde lo empresarialy lo sostenible, no solo el Estado y la Sociedad Civil deben ponerse de acuerdo, también los actores privados, ello implica que una de las múltiples formas de diálogo que deban darse desde los territorios sea que las empresas comprendan que aportar a la paz va más allá de la generación de empleo y del pago de impuestos, que reconozcan que la competitividad y sostenibilidad de los negocios dependen del bienestar de sus entornos y de las personas, sean clientes y consumidores, trabajadores y proveedores o comunidad beneficiada, que respeten las instituciones sociales, económicas, políticas y culturales presentes en su área de influencia, complementando las estrategias e intervenciones de estas, en vez de reemplazarlas, entendiendo que ello es una inversión a largo plazo y tomando decisiones que benefician el desarrollo socioeconómico sostenible de sus áreas de influencia tanto en el presente como en el futuro, los sectores económicos, el minero, el sector agrario, pecuario, el turismo, los servicios, entre otros.

En materia ambiental, por ejemplo, al mismo tiempo que la guerra impidió el desarrollo para la economía, una cantidad de territorios se fueron expandiendo modelos extractivitas que hoy con mucho análisis muestran que creómuchas dificultades, exclusiones, nuevascondiciones de pobreza, nuevas modalidades de desplazamiento de los territorios, el segundo es el tipo de 
economía que queremos dialogar, el tipo de aparato productivo más diverso de inclusión de una producción popular, comunitaria que fue afectada por la guerra.

La propuesta de realizar diálogos entre sectores que son opuestos permitirá respecto a las posturas sobre el modelo de desarrollo, los usos del suelo y los territorios que cada parte tenga. Un punto de equilibrio que permita a las empresas y al Estado proponer un modelo que no esté aislado de la visón colectiva de los habitantes del territorio, que no lesione sus intereses, su cultura, su cosmovisión, dejar a un lado la Dicotomía "Desarrollo o Conservación ambiental", es posible una tercera vía, sentar en la mesa a actores estratégicos a dialogar, pasando por la fase de: Primero dialogar, en lugar de monologar, luego, identificar esos grandes puntos de tensión, en los que no es posible que se lleguen a acuerdos, en tercer orden, proponer soluciones a los problemas y con ello finalmente se descubriría que todos, por muy opuestos que sean tienen una visión común, todos quieren un desarrollo económico y social, aumentar su productividad, empoderarse, generar riqueza, desdés perspectivas y roles distintos, quieren la conservación del medio ambiente, logrando rediseñar un modelo sostenible con enfoque territorial.

Colombia se enfrenta a un nuevo reto, eso incluye afrontar a lo inimaginable, superar el dolor de manera transformadora, hay que transicionalizar a Colombia, en todas sus esferas, sentar a las comunidades y lograr métodos de hacer vinculantes esos acuerdos, eso requiere voluntad política y empresarial, empoderando a las comunidades para generar la sostenibilidad de los acuerdos que ellos mismos puedan generar, por ello son importantes instituciones como la Agencia Nacional de Desarrollo que impulsa los Programas de Desarrollo con enfoque territorial, como los Programas de Desarrollo y paz, que funcionan con fondos privados, este tipo de escenarios son prueba de que el diálogo es el camino para transformar positivamente sociedades divididas a través de un proceso de diálogo. 


\section{La importancia de los PDET en el desarrollo territorial, el progreso empresarial y de las comunidades.}

A través de los PDET (Programas de Desarrollo con Enfoque Territorial) se puede incidir positivamente para que estos generen oportunidades de cambio y desarrollo. Estos han sido diseñados a 10 años, cumplen un papel central en la Construcción de paz en los territorios, el objetivo es generar espacios de diálogo social sobre temas trascendentales como el ordenamiento y uso del suelo, reactivación económica, vivienda, salud, educación, infraestructura, entre otros, todos estos son aspectos relacionados con el desarrollo.

En las mesas de diálogo veredales, municipales y subregionales se encuentran representantes de las entidades territoriales, autoridades étnicas, comunidades, organizaciones sociales, comunales y comunitarias y el sector privado. Esta es una política que sin duda alguna nace de la filosofía de la participación democrática, estos acuerdos están blindados legislativamente para tener efectos vinculantes a través del Acto Legislativo 01 de 2016, el cual facultó al Presidente de manera excepcional y extraordinaria a expedir decretos con fuerza material de ley orientadas a la implementación del Acuerdo Final y las sentencias C-699 de 2016, C-160 y C174 de 2017 dándole validez constitucional a los decretos leyes, haciéndolos obligatorios, dada su trascendencia e importancia para el Estado Social de Derecho.

\section{Conclusión}

A modo de conclusión es de gran importancia resaltar que Financieramente, también se ha generado una sostenibilidad de ese diálogo, para ello se han dispuesto recursos del Presupuesto general de la Nación, del sistema general de participaciones, del de regalías, así como otros recursos de cooperación internacional y empresas privadas que le apuestan a este proceso, ello es sumamente importante, porque permite combatir la famosa "fatiga del diálogo".

Por lo anterior, una tercera esfera de garantía debe ser el aporte de la sociedad civil, debe haber un cambio de mentalidad de la forma en cómo funciona el mundo territorial, eso implica veedurías, generar centros de pensamiento,gruposdediálogoyconvivencia, unasociedad civilmínimamente 
organizada, que frene los procesos de corrupción empoderándose de sus procesos, las inversiones donde no existe priorización de gasto a partir de la participación directa en la toma de decisiones, eso se logra en una última fase, cuando se ha entendido lo que implica la democracia y luego, cuando se ha comprendido la utilidad del diálogo en el diagnóstico de problemas y sus soluciones, pero también como un espacio de sensibilización transformadora, que incluso llega a transicionalizar los intereses apostando todos a generar condiciones dignas de vida, entendiendo que sí uno crece, crecen todos.

Las empresas también participan en esta dinámica y lo saben. El mercado se fortalece en cuanto los consumidores tienen poder adquisitivo, y ello es posible si hay formas de producción, que deben ser amigables con el medio ambiente, por eso las comunidades indígenas y afro descendientes son importantes en estos diálogos territoriales.

Entender que todo escenario que lleve a la consolidación del histórico acuerdo de paz en el país y en los territorios, desde sus dinámicas específicas en todos los ámbitos imaginados o por imaginar empiezan necesariamente por un diálogo entre opuestos, es la clave de la transformación social que necesita el País, el modelo de productividad y competitividad del sector empresarial y productivo no será sostenible, sí no se trabaja desde las dimensiones o dinámicas comunitarias y se proponen nuevas herramientas de co creación y co gobernanza, un sistema propuesto desde los PDET.

Ya se están realizando algunos diálogos en el País desde los territorios y con actores estratégicos, promovidos por la misma institucionalidad y otros por iniciativa privada, pero además de eso, hay otros espacios de diálogo que deben generarse que deben nacer desde la sociedad civil, y que tienen como reto generar la sostenibilidad de este nuevo proceso, pero sobretodo de llegar a consensos que todos promuevan y defiendan, es la era en la que una gran masa crítica se prepara para encontrar en la democracia participativa una forma de vida. 


\section{Referencias}

Bauman, Zygmunt (2007). Tiempos Líquidos. Recuperado de https://catedratesv.files.wordpress.com/2016/07/bauman-zygmunt-tiempos-liquidos.pdf

De Roux, Francisco (2017, 25 de enero). Diálogo entre opuestos. El Tiempo. Recuperado de http://www.eltiempo.com/opinion/ columnistas/francisco-de-roux/dialogo-entre-opuestos-francisco-de-roux-columna-el-tiempo-53631

Diez, Elena. (2018). Experiencias internacionales, Guatemala. Alto comisionado para la paz, Diálogos entre Opuestos, en procesos de transición. Conversatorio, Bogotá, Colombia.

Espiau, Gorka. (2018). Experiencias internacionales, País Vasco. Alto comisionado para la paz, Diálogos entre Opuestos, en procesos de transición. Conversatorio, Bogotá, Colombia.

Ibáñez, Lina. (2018). Debate sobre tensiones en el desarrollo. Alto comisionado para la paz, Diálogos entre Opuestos, en procesos de transición. Conversatorio, Bogotá, Colombia.

Lévinas, E. (1990). De otro modo que ser o más allá de la esencia. Paris: la librería de Poche.

Organización de las Naciones Unidas. (2004). El Estado de derecho y la justicia de transición en las sociedades que sufren o han sufrido conflictos. (S/2004/616). Recuperado de https://undocs.org/ $\mathrm{es} / \mathrm{S} / 2004 / 616$

República de Colombia. Congreso de la República. Acto legislativo 01 de 2016.

República de Colombia. Corte Constitucional. Sentencia C- 160 de 2017. Magistrado Ponente: Gloria Estella Ortiz Delgado.

República de Colombia. Corte Constitucional. Sentencia C- 174 de 2017. Magistrado Ponente: María Victoria Calle Correa.

República de Colombia. Corte Constitucional. Sentencia C- 699 de 2016. Magistrado Ponente: María Victoria Calle Correa.

República de Colombia. Ministerio del Interior. Decreto 893 de 2016.

Revel, Jean François. (1983). Cómo terminan las democracias. Barcelona. Editorial Planeta S.A.

PDET (Programas de Desarrollo con Enfoque Territorial)-Colombia 2018. 